\title{
Desarrollo rural, política y capacidades desde el Modelo de Organización. Caso de tres grupos del sur de Costa Rica
}

\section{ROY ATENCIO MORALES}

> Universidad Nacional, Campus Coto, Neilly - Paso Canoas, Costa Rica. ratencio3@yahoo.es

Universidad de Valparaíso

Facultad de Arquitectura

Revista Márgenes

Espacio Arte Sociedad

Desarrollo rural, política y capacidades desde el Modelo de

Organización. Caso de tres grupos del sur de Costa Rica

Diciembre 2015 Vol. 12 № 17

Páginas 62 a 68

ISSN elec. 0719-4463

ISSN imp. 0718-4034

Recepción: septiembre 2015

Aceptación: noviembre 2015

\section{RESUMEN}

El siguiente artículo es el resultado de un proyecto desarrollado durante los años 2013 y 2014 por el equipo de extensión de la Sede Coto y SIMPE de la Universidad Nacional de Costa Rica con organizaciones de Cantones del sur de este país.

La dicotomía de la distribución de la tierra en la zona sur de Costa Rica da pie a múltiples factores que confluyen en una distribución de los ingresos muy diferenciados, donde no toda la población tiene la oportunidad de ingresar a mejores niveles de ingresos y accesos a mejores condiciones de calidad de vida.

Lo contradictorio de la situación es que la zona cuenta con cantidad y calidad de recursos naturales que harían en otras latitudes una zona ganadora, tanto para el desarrollo regional, como para el desarrollo local, sin embargo, éste es un criterio encontrado con singularidad hacia grupos muy pobres y un pequeño grupo de ricos, así como capital transnacional que explota las riquezas de la zona, dejando sólo los ingresos de bajos salarios y algunos programas sociales, los cuales compiten con las funciones del Estado y las instituciones Estatales locales.

El desarrollo implica la relación de algunas variables, entre ellas la existencia de capital de inversión y capacidad de gestión de las personas, así como de grupos organizados. A la par de esto existe capital financiero suficiente, no sólo en la banca nacional y privada, sino en las instituciones del Estado. Se contradice lo anterior de la conjunción de tres variables para el desarrollo (capital, organización, y riqueza).

PALABRAS CLAVES

desarrollo rural, políticas, capacidades de gestión, tipos de organización

Rural development, policies and capacities from the Organization Model. Three case groups in southern Costa Rica ABSTRACT

The dichotomy of the distribution of land in the southern zone of Costa Rica gives rise to multiple factors that come together in a distribution of highly differentiated income, where not all people have the opportunity to enter higher income levels and access the best quality of life conditions.

The contradictory nature of the situation is that the area has a great quantity and quality of natural resources that would in other latitudes turn the region into a winning area, both for regional and local development. However, this is a criterion met only by groups of very poor inhabitants and a small group of wealthy people and transnational capital that exploits the riches of the area, leaving only the income of low wages and some social programs which compete with the functions of state and local government institutions.

The development involves the relationship of some variables, including the existence of investment capital and management capacity of individuals and organized groups. Along with this, there is sufficient financial capital, not only in national and private banks, but also in state institutions. The conjunction of three variables for development (capital, organization, and wealth) contradicts the ideas exposed above.

KEYWORDS

rural development, policies, management capacity, types of organization 


\section{SOBRE EL DESARROLLO TERRITORIAL Y POLITICA}

Se debe aclarar que se va a entender como desarrollo territorial y política como conceptos necesarios para interpretar los cambios y el origen de los detonantes al cambio. Si el desarrollo lo interpretamos como:

un proceso multidimensional que implica cambios en las conductas y actitudes individuales que impactan las estructuras institucionales de los sistemas económicos, sociales y políticos cuya finalidad es la persecución del crecimiento económico y el desarrollo social mediante la reducción de la desigualdad y la reducción de la pobreza (Cueva J., 2013:1).

Según José Guadalupe Vargas Hernández (2007:110), El desarrollo es la condición de satisfacción de las necesidades de la población de una nación mediante el uso racional y sustentable de los recursos naturales. "El desarrollo es la satisfacción de necesidades de unidades como el individuo, el pueblo, la ciudad o el grupo social identificado por factores tales como el origen étnico, clase, género, sexualidad o edad" (Sutcliffe, 1995:45, mencionado por Hernández, 2007), a estas palabras se deben de agregar las posibilidades reales para que un proyecto social económico y ambiental se haga una realidad.

El desarrollo es pues sustentado en cuatro polos de crecimiento 1. Las pequeñas y medianas empresas (PYMES);

2. Las corporaciones transnacionales y nacionales (CTN); 3. Las cooperativas y, 4. Las empresas e instituciones estratégicas del Estado (Vargas, 2007:121), con un modelo de acercamiento de varios subniveles que son los que determinan la misma sociedad. Donde la solución de problemas es uno de los principios de la lógica del desarrollo.

La sinergia de estos cuatro pilares sustentan el desarrollo como política al conjunto de decisiones interrelacionadas tomadas por actores políticos preocupados, seleccionando metas y los medios para alcanzarlas en una situación determinada, por todas las organizaciones, que se ven representados en esos espacios políticos.

Por Política Gubernamental se va a entender una política elaborada por el gobierno y entendida como Política Pública una práctica social y no un evento aislado, ocasionado por la necesidad de reconciliar demandas conflictivas o establecer incentivos de acción colectiva entre aquellos que comparten metas.

Entendemos, como Política regional a la intención gubernamental explicita en el sentido de afectar, con su acción, una parte determinada del territorio nacional, y la determinación de los objetivos y metas que se propone alcanzar en un plazo establecido (Ornelas, 1993, citado por Carlos Hernández Medina p. 3).

\section{CAMBIOS Y SECTOR AGRÍCOLA}

Con los problemas del cambio de precios del café muchos lugareños se dedicaron a la siembra hortícola, ya que este tipo de producción genera condiciones de excelente productividad por área y para una pequeña granja de 4 ó 5 hectáreas este tipo de producción se veía como alternativa rentable. Algunos productores de café que poseen pequeñas áreas de tierra vieron en la producción de verduras una tabla de salvación a las ya de por sí condiciones precarias que venían teniendo con el café. Sin embargo, el cambio les generó otros problemas que ellos no habían tomado en cuenta.

En el sector agrícola el paradigma de la globalización ha implicado un cambio de orientación en las decisiones de los productores, impulsado tanto por fenómenos externos, como por la manera en la que los Estados Nacionales, las regiones y los propios actores involucrados tratan de responder a ellos, realizando cambios en los sistemas de producción para enfrentar las demandas del mercado.

En este caso no existía una relación vertical entre productores y comercializadoras de manera que el productor tuvo que enfrentar el mercado local en condiciones que otros no lo hacen, con la consecuencia de que muchas veces su producto no es aceptado, ya que no existía respaldo de contrato. Además de que los grandes conglomerados dominan el mercado agroalimentarios transnacional, quienes aglutinan producción: en cantidad, calidad y variedad para comercializar durante todo el año a través de diversos mecanismos de integración con productores de todo el mundo. Este negocio que se ha formado de esta manera deja desprotegido al pequeño y mediano productor, si no está alineado a estos conglomerados.

Uno de los mecanismos sobre los cuales el pequeño y mediano productor trató de basar su producción para la comercialización ha sido el contrato, que garantiza al productor que una empresa va a comprar toda su cosecha, y garantiza un precio sobre el cual el productor realiza sus cálculos de pérdidas o ganancias, así como sus flujos de caja para operar durante la producción.

Lo anterior se transforma en un debilitamiento de los productores individuales, así como de los territorios particulares desligados de estos conglomerados. Si los productores no se organizan formalmente no son capaces de enfrentar a estos conglomerados productores no sólo dentro del país sino fuera de éste.

El consumidor hoy es más exigente, conoce, tiene claro que es calidad, y necesita saber las condiciones en que los productos que va a consumir se producen. El agricultor debe pasar de ser un productor de subsistencia a ser un productor empresarial, con conocimiento de sus clientes finales, de sus compradores locales, nacionales e internacionales y decidir en qué tipo de mercado participar, en este caso los productores deciden participar en el mercado local inicialmente.

Implica entonces que el agricultor debe de formalizar redes que en forma conjunta se fortalezcan para poder ingresar a los nuevos sistemas agroindustriales; articulándose territorialmente, pues los procesos de producción, distribución, comercialización y consumo de las mercancías, son realizadas por actores localizados en distintas zonas geográficas, y como productores individuales son incapaces de poder realizar todos los sistemas desde la producción hasta el consumo final.

Lo anterior no sólo demuestra las debilidades de los productores individuales ante el comercio internacional, y hasta local, como la necesidad de transformación de la agricultura regional e individual, en términos de producir para mercados cada día más sofisticados y exigentes, sino en los cambios de productividad por área y por ciclos de manera que el mercado esté siempre abastecido. La agricultura científica sustituye la agricultura cultural, tradicional, de subsistencia y focalizada para mercados locales menos exigentes. Ello conlleva a una participación de un mercado que paga menos por el mismo producto, y ello se transforma en menos ingresos para los productores locales.

La agricultura científica implica la inversión en tecnología, muchas veces la cual no está disponible a nivel local, lo que se transforma en un elemento más de imposibilidad para actores locales, de participar en ese nuevo sistema productivo, implica vulnerabilidad de estos actores sociales, sobre los cuales la política nacional debe de 
actuar para hacer posible que estas personas no sean dejadas de lado en el nuevo contexto de producción. Lo anterior se transforma en más erosión de la riqueza de muchos, incapaces de enfrentar por sí solos este nuevo esquema agrícola. Es aquí donde el Estado es Ilamado a ser solidario y participar con éstos para fortalecer sus necesidades y que sean capaces de enfrentar en iguales condiciones al mercado que el mismo se ha encargado de fortalecer.

El desafío es pues una agricultura que industrialice los procesos, de manera que los agricultores tradicionales den el salto a una agricultura industrial, que pueda enfrentar cualquier mercado local o internacional, sin mayores problemas.

Los mercados han creado en los agricultores la idea que la vida del campo no es económicamente rentable, ya que las grandes empresas producen todo más barato y estandarizado, de manera que se debe de migrar a los centros urbanos para mejorar los niveles de vida, buscar un empleo y que sus hijos tengan un mejor futuro.

Además el mercado se encarga de hacer creer que los productos traídos desde los países que se han especializado en la producción agropecuaria son mejores y más baratos que los producidos en el mismo país. Constantemente se ve a los productores nacionales quejarse de sus problemas de precios, de productividad, de los efectos negativos de los cambios climáticos, de las malas vías de comunicación y de los efectos negativos de los mercados nacionales, por el efecto de los intermediarios.

La agricultura no puede realizar cambios si no basa su producción enfocado en un proceso industrial, competitivo, con un concepto de estandarización, y una productividad creciente en cada ciclo. Los controles de eficiencia y uso tecnológico son base primordial para que el agro realice un cambio de fondo. $Y$ el agricultor nacional tenga en sus manos un proceso de acumulación, capaz de transformar en una creciente inversión productiva.

La claridad está enfocada en términos que el mercado no hace cambios las exigencias de los productos que consumen, si no que espera que los productos mejoren constantemente, y que los precios no varíen mucho. La producción agropecuaria con la idea de un proceso industrial. El productor no puede por sí solo enfrentar estas condiciones.

En términos de seguridad alimentaria, las familias cambian sus requerimientos alimentarios, ello hace que los agricultores nacionales sean los primeros en conocer las necesidades de los consumidores finales y poder hacer los cambios desde la producción; no lo contrario como ha venido pasando, que son las necesidades de los consumidores los que han hecho que el agricultor cambie sus sistemas productivos. El agricultor no puede esperar que los gustos y preferencias del consumidor varíen para él adaptarse, debe de ser capaz de ofrecer nuevos productos. Sólo un pensamiento industrializado, a la par de innovación, tecnología e investigación de la mano pueden hacer que este nuevo proceso ocurra.

Al lado de un cambio productivo está el cambio de las políticas desde el Estado, con la creación de toda la infraestructura necesaria para que el nuevo modelo se implemente. La nueva ruralidad inicia con claridad de políticas desde el Estado y con cambios de mentalidad de los agricultores.

Los problemas de largo plazo requieren nuevas aproximaciones y soluciones innovadoras. Los retos globales se encuentran relacionados, las prioridades y elecciones tendrán repercusiones de largo alcance. Todo lo anterior tiene que ver con las causas y efectos de la pobreza: falta de ingresos, falta de acceso al agua potable y saneamiento básico, cuidado de la salud, alimentación y vivienda. Indudablemente, la clave para reducir la pobreza se encuentra en la creación de bienestar. Los negocios proveen el impulso para el crecimiento económico; el sector privado puede dar soluciones duraderas para el desarrollo, junto con la generación de prosperidad y oportunidades. La creación de oportunidades es la clave para reducir la pobreza,

Las empresas no pueden ser exitosas en sociedades que fallan. De la misma manera, donde las empresas son reprimidas, las sociedades fallan en prosperar (Björn Stigson, Presidente Fundación Shell WBCSD:12).

Lo anterior se transforma en nuevos requerimientos desde los municipios de manera que sean éstos los llamados a co-generar el desarrollo local desde su plataforma de acción territorial. Los municipios deben en sí mismos hacer un cambio hacia la persecución de la prosperidad local, de su territorio. Ello implica hacer alianzas con los poderosos y poder traspasar las ganancias de éstas a los que menos poseen en su ruralidad. Los municipios rurales deben de enfocar sus baterías a golpear aquellas barreras que dificultan la productividad, el comercio, el transporte, y la comunicación local, de manera que la información sea parte cotidiana de todo productor.

En teoría, en la práctica y en términos del sentido común... la mayoría de los caminos para salir de la pobreza comienzan en las empresas (Kurt Hoffman, Director Fundación Shell WBCSD:15).

Las ciudades abren nuevos espacios para nuevos negocios, y con ellas nuevas familias inician sus aventuras empresariales en la ciudad, pero no todas las familias pueden y deben de estar en las ciudades, el campo necesita un cambio de mentalidad, donde los municipios les corresponde cogenerar oportunidades, mejorando vías de comunicación, la nueva ruralidad se enfoca más en poder sostener la población del campo en el campo, con condiciones de vida cada vez mejores. Las fincas deben de ser capaces de ser autosuficientes en la generación de ingresos familiares para que esta población pueda acceder las mismas condiciones que aquellas que viven en las ciudades, evitando así las migraciones campo ciudad.

El Desarrollo Rural Integrado (DRI) evolucionó introduciendo el componente de vías de comunicación, servicios básicos de salud, educación y electrificación y promoción de la organización y participación de la comunidad. Se consideraron indispensables un mayor esfuerzo y participación de los gobiernos locales, una mejor coordinación de las entidades comprometidas y, lo que es más importante, un mayor interés y participación de los productores.

De este modo, el desarrollo comienza a ser visto como un conjunto de capacidades generadas endógenamente, ligadas a la calidad de los recursos humanos, la disposición organizativa de los agentes, la creación de entornos adecuados a diferentes fines (inversión, residencia, turismo, innovación), la calidad institucional territorial, las diversas formas de cooperación y aprendizaje, los mecanismos de resolución de conflictos, la coordinación de políticas económico-sociales. Cuando esas capacidades endógenas se expresan, permiten que los impulsos exógenos se inserten en un sistema territorial organizado.

No puede haber desligamiento de lo político con lo local, ya que es el Estado a través de sus instituciones las llamadas a acompañar al 
cambio de paradigma productivo, de los mercados y a hacer las inversiones en las cuales la empresa privada no tiene espacios claros de inversión. El Estado acompaña a la empresa privada en la cogeneración del nuevo modelo productivo. De ahí la responsabilidad de los territorios de organizarse, de iniciar los cambios desde las bases productivas, comerciales y del conocimiento para lograr en un corto plazo la nueva ruralidad local y las nuevas oportunidades comerciales productivas que enmarcan el desarrollo y la prosperidad.

\section{RESPONSABILIDAD DEL GOBIERNO LOCAL EN EL DESARROLLO}

La capacidad de respuesta de los gobiernos locales está asociada a las capacidades de formulación e implantación de políticas públicas que promuevan el desarrollo económico mediante un incremento de las contribuciones de las actividades económicas, políticas y sociales. Desafortunadamente, los gobiernos locales no reconocen su responsabilidad como agentes del desarrollo local en la toma de decisiones relacionada con la sociedad. Los gobiernos locales constituyen las instancias más próximas a la participación de la ciudadanía y tienen una función importante como agentes del desarrollo económico y social de las localidades en la dinámica de una economía globalizada.

El gobierno local es el llamado a generar los espacios de toda índole, donde la sociedad local se vea potenciada a una mejora continua de sus actividades, de su producción, de su comercialización y hasta de su zona como especial para hacer vida familiar.

Entre las principales funciones del gobierno local está la de detectar y crear los espacios de interacción y contacto entre los actores y sus mecanismos de negociación, resolución de conflictos y procesos de toma de decisiones, la selección de opciones de políticas públicas y la implantación de proyectos de desarrollo local (Hernández, 2007:124).

Direccionar las estrategias para el desarrollo local, guiadas por una fluida acción del desarrollo de corto y largo plazo en el territorio, se divide en diferentes niveles de acción con metas claras para el logro. (Hernández, 2007:125) La estrategia de crecimiento económico se orienta hacia el desarrollo local basado en los proyectos municipales impulsados por los agentes económicos y actores políticos y sociales locales.

El ejercicio de planificación debe venir asociado con las políticas de fomento productivo y los instrumentos de financiamiento de las iniciativas locales. Sólo con el surgimiento de instituciones flexibles de financiamiento, la integración entre los agentes públicos y privados y la innovación en las formas de gestión y organización de los emprendimientos se generan condiciones para financiar y apoyar el desarrollo de los pequeños emprendedores y de experiencia de producción comunitaria.

\section{ACERCAMIENTO METODOLÓGICO}

Metodológicamente, el resultado de este artículo está en función de un proyecto de acompañamiento a las organizaciones que se desarrolló en los años 2012 y 2103. Por el equipo de trabajo conjunto entre la Sede Coto y el CINPE, (unidades que pertenecen a diferentes sedes de la Universidad Nacional) y se incorporaron de estudiantes de la sede Coto como participantes en el proyecto.

Para la determinación de MIPYMES (micro y pequeñas empresas), $\mathrm{u}$ organizaciones beneficiaras del proyecto se partió de una con- sulta con actores locales relevantes, tales como Municipalidades, Ministerio de Agricultura, Ministerio de Economía, IDA, (instituto de desarrollo agrario, hoy INDER), CNP (consejo nacional de la producción), entre otros que trabajan con organizaciones locales y poseen las listas de contactos de cada una de ellas, listas que fueron utilizadas para seleccionar una muestra para ser visitadas previamente. Una vez lograda esta etapa, se realizó una visita a la muestra de organizaciones, con el objetivo de aplicar los diagnósticos previamente construidos, y de esta forma evaluar el estado actual de cada una de ellas y su potencial futuro.

Los resultados del diagnóstico fueron analizados para seleccionar con base en criterios objetivos (inversión, infraestructura, liderazgo), las organizaciones que serían las beneficiarias del proyecto. A partir de esta definición, se comenzó un proceso de apoyo a 3 organizaciones (las cuales no sólo representan a otras organizaciones, sino que poseen la experiencia y recursos necesarios para agendar los trabajos que resultaron a partir del diagnóstico y se planteó una propuesta piloto. Resulta claro que temas tales como fortalecimiento organizativo, gestión financiera, conocimiento del mercado y planes de negocios, serán aspectos claves que se desarrollarán durante el proceso de acompañamiento, que se ejecutó durante un período de 16 meses.

Para los casos que así lo ameritaron, se apoyó en la construcción de planes de negocios que permitieran acceder a créditos o financiamientos por las diversas instancias existentes. Se buscó propiciar el acercamiento entre las organizaciones beneficiarias, las entidades financieras y los estudiantes eran los encargados de estas funciones. Se trabajó con 18 estudiantes, durante todos los 16 meses, a 6 estudiantes en cada semestre, los cuales cursan el 4to años de las distintas carreras de las Sede Coto, Universidad Nacional, (Turismo, Administración e Ingeniería), quienes en su práctica supervisada acompañaron a las organizaciones a realizar los documentos que así ameritaban para acceder otros niveles económicos en dichas organizaciones (estudios financieros, estudios de factibilidad, planes de negocios, brochures informativos, guías turísticas, planes estratégicos, talleres de formación, etc.).

Para la recolección de datos se utilizaron diferentes cuestionarios, así como visitas y trabajos con representantes de cada organización en sitio, algunos por horas y otras veces por días.

Los resultados del proceso de acompañamiento y de generación de capacidades se expusieron en un taller a cada una de las organizaciones participantes y público en general, de cada uno de los lugares donde se trabajó, de manera que se pueda generar una visón de problemática y los posibles caminos para dar soluciones particulares. Esto implicó documentar los resultados de cada objetivo, para poder devolver los resultados a los participantes, quienes serán al final los más beneficiados al tener clarificadas sus fortalezas y debilidades y sus posibilidades como emprendedores comerciales productivos.

De forma paralela al trabajo con las organizaciones beneficiarias, se desarrollaron 2 talleres con aquellas organizaciones que no fueron seleccionadas como prioritarias dentro del proyecto. Estos talleres incluyeron los temas de fortalecimiento organizacional, gestión financiera y conocimiento de mercados y estrategias de mercadeo. Se contó con más de 30 participantes en cada taller, los cuales pertenecían a diferentes organizaciones locales. 
Particularmente, se consideraron recursos para transporte a lo interno, viáticos, actividades de capacitación, labores de impresión, becas a estudiantes y equipo. Al ser un proceso intensivo en giras de campo, combinando el equipo de trabajo del Campus Coto y del CINPE, este componente presupuestario asume una alta relevancia en el presupuesto total del proyecto.

\section{DESDE EL CAMBIO DE MODELO Y POLÍTICA NACIONAL}

En los últimos 30 años el país ha sufrido cambios drásticos en las propuestas del modelo rural desarrollado. Los cambios de política que se han generado a lo largo de estos años, han reflejado las políticas de los países desarrollados y los organismos de financiamiento mundial, llevando el desarrollo rural hacia la desprotección, el libre mercado, y la competencia no sólo interna sino internacional, vía la libre entrada de productos externos, los cuales compiten en los mercados nacionales contra los producidos internamente. Ello ha llevado a una paulatina desprotección de las organizaciones y el productor nacional desde la política, y desde las instituciones, que en su momento fueron creadas para fortalecer y proteger la producción nacional. En sus años el gobierno animó a la conquista de nuevos territorios y el crecimiento de la frontera agrícola, a la dependencia casi en su totalidad de la producción agrícola, luego con ayuda de la banca nacional forzó el modelo de sustitución de importaciones, la industrialización interna, y la migración campo ciudad para tener disposición de mano de obra. A su vez este modelo crea condiciones para que el agro produzca materia prima necesaria en dicho modelo para algunos productos.

Lo anterior no sólo creó condiciones viables para el productor nacional, sino que crea el mercado necesario, fortalecido desde el Estado, no sólo provee instituciones, sino facilita los cambios y las transferencias tecnológicas, desde los países desarrollados hacia el agro y la industria nacional. Y en este mismo tiempo inician la entrada de grandes transnacionales al modelo de producción nacionales, financiados con capital internacional, más fuerte y solvente que la misma banca nacional, igualmente la banca nacional financia ciertas empresas transnacionales, dadas estas capacidades.

De un proceso de expansión de fronteras agrícolas a un proceso de formalización de la posesión de las tierras, a un nuevo modelo de crecimiento buscando los mercados internacionales con la producción nacional. El cual pronto es visto desde las transnacionales como una nueva forma de acumulación, migrando el negocio hasta la producción, y los estados ven en ello una fortaleza, pues se garantizan con estas compañías el empleo, y el desarrollo local, tanto que en algunos de estos enclaves, estas compañías sustituyen al mismo el Estado en la construcción de la infraestructura. Específicamente esto genera una dicotomía de la distribución de la tierra en la zona sur (Costa Rica), da pie a múltiples factores que confluyen en una distribución de los ingresos muy diferenciados.

Lo contradictorio de la situación es que estas zonas cuentan con cantidad y calidad de recursos naturales que harían en otras latitudes una zona ganadora, tanto para el desarrollo regional, como para el desarrollo local, sin embargo existen criterios encontrados, con la singularidad hacia grupos muy pobres y un pequeño grupo de ricos, así como capital transnacional que explota las riquezas de las zonas, dejando sólo los ingresos de bajos salarios y algunos programas sociales.

\section{DESDE LOS MUNICIPIOS}

En estos términos el problema que muchos municipios ven en su población campesina o rural, no es más que una ventaja, y una oportunidad para generar nuevos paradigmas productivos en la nueva ruralidad. Lo mejor no es que exista una migración constante de un Cantón, que tienen las posibilidades de ser uno de los más activos en las economías locales, sino más bien dar un vuelco a la producción local y explotar esos espacios productivos para los que el territorio tiene fortalezas.

El proceso de globalización se encarna en las ciudades y se trasfiere al campo por medio de la producción para mercado, mercado cada vez más competitivo, el cual busca cada día más calidad, y a menores precios, condición sobre la cual el productor o cambia o perece. Sin embargo el Estado es llamado a través de sus instituciones a ayudar a aquellos que menos tienen a que el cambio se haga factible.

Estaría por demás mencionar que la sociedad organizada cambia la estructura de poder y de decisión, no para enfrentar al Estado y sus instituciones, sino porque no confía más en los políticos locales, que ve en ellos la imposibilidad de generar espacios para el desarrollo local, y ve en la organización de la sociedad civil una oportunidad para romper con los paradigmas tradicionales institucionalistas y crear sus propios conceptos del desarrollo local y territorial, donde se le den oportunidades a los grupos de enfrentar las condiciones de mercado a su propio ritmo.

Y el control local del proceso de desarrollo se verá facilitado si existe un clima social de autoconfianza entre actores y un permanente cambio y adaptación de las instituciones. El nuevo paradigma es así un instrumento potenciado desde los municipios el cual posee un poder aún no visto por sus alcaldes, al hablar de territorialidad. La alcaldía local es llamada a realizar los esfuerzos de cambio en su gestión, no sólo para cobrar bien sus impuestos sino para saber dónde y cuándo gastarlos en su territorio, de manera que afecte en lo más posible a la población sobre la cual está posesionada como gobierno local.

Los cantones rurales deben de fortalecer cada sector que ayude a generar ingresos redistribuidos localmente, es una carrera contra el tiempo y contra el mercado, así como contra la producción tradicional.

\section{CONCLUSIONES RELEVANTES}

Las conclusiones del proyecto se estructuraron de acuerdo a los objetivos específicos del proyecto:

\section{Sobre el marco administrativo y productivo}

1. El grado de avance de las organizaciones en el lapso del proyecto fue reducido. La realidad indicó que las organizaciones sí requieren capacitarse, pero sólo en el corto plazo ya que los resultados los esperan de forma casi inmediata; lo cual no es posible en un proceso de creación de capacidades competitivas y de desarrollo local.

2. El proyecto contribuye a identificar necesidades en materia de gestión empresarial y competitividad, pero éstas son muy heterogéneas en comparación a los temas que cubre el proyecto. Además, el grado de complejidad de asuntos como la comercialización de hortalizas en Coto Brus, ha demandado y demandará acciones mancomunadas del sector público y privado que escapan el alcance de esta iniciativa. 
3. Sobre el estado de gestión y capacidades de las organizaciones: A pesar de haber realizado un proceso exhaustivo de revisión y selección de organizaciones en base a criterios claves, como liderazgo, capacidades de gestión e infraestructura, que permitiera avances más rápidos en los beneficiarios, la realidad de las organizaciones demostró lo contrario. Las MIPYMES y las organizaciones base están urgidas de apoyo pero no en materia de capacitación, sino han sido ayudadas desde el asistencialismo, lo cual se ha reproducido por décadas, mermado en la metodología de trabajos de las instituciones estatales y de algunas fuentes de cooperación técnica, y las han inmerso en una forma de organización con miedos de enfrentar las fuerzas del mercado en forma propia.

4. Metodología de selección: los supuestos sobre los que se seleccionaron las organizaciones no dan los resultados esperados. El trinomio inversión, capacitación y liderazgo no resultó en una mejor capacidad de gestión de las organizaciones base. Pues el resultado de cambio está más allá de dos años que dura el proyecto. Se necesita un proceso de más número de años hasta que cambien de mentalidad y capacidades.

5. El trabajo con Catubrus, (Cámara de Turismo de Coto Brus), como estudio de caso y de extensión es interesante en el sentido de que se escapan del modelo asistencialista. Se trata de una figura compuesta en su mayoría por jóvenes profesionales, que aun sin recursos económicos han emprendido acciones de planificación del sector turístico por medio de una visión de largo plazo. CATUBRUS tampoco se encuentra amparada en las figuras organizativas tradicionales en la región (Leyes 218 y 3859, de Costa Rica). Ello da luces para argumentar que más allá de los recursos económicos, impulsar iniciativas emprendedoras y de impacto en las regiones es un tema de recursos humanos y gestión de capacidades con una visión futura.

\section{Diseñar Plan Piloto}

1. A razón de que cada organización tiene sus capacidades específicas y sus necesidades, no se puede desarrollar un plan piloto general. Al momento de plantear el proyecto se esperaba una capacitación general pero eso no fue posible porque se tenían sujetos diversos cuyos proyectos productivos se encontraban en diferentes etapas. Esto da como resultado de crear una propuesta del plan piloto de las organizaciones y no su implementación. Lo anterior demuestra que las organizaciones si bien requieren capacitación y acompañamiento, están más urgidas de un mejor recurso humano, acceso al crédito y recursos semillas, para poder migrar del asistencialismo Estatal a empresa.

2. En el caso de Catubrus, se logra validar el plan piloto a través de la socialización del Plan Estratégico de este ente.

\section{Desarrollo de capacidades empresarial y planes de negocios}

1. Las organizaciones no tienen la capacidad administrativa ni de gestión para darle seguimiento al proceso de fortalecimiento empresarial. Por ellos mismos no son capaces de hacer los procesos de gestión administrativa desde la organización para dotar a los proyectos de una visión de negocios. No quieren asumir riesgos de inversión en función de la organización.
2. Se desarrollan exitosamente los planes de negocios, cuyos resultados fueron positivos para la idea de negocio. No obstante, las organizaciones carecen de capacidades para su implementación y seguimiento.

3. Nuevamente Catubrus marcó la diferencia en materia de desarrollo de capacidades empresariales, pues dan un giro en su visión inicial de asociación, por ello, se da una evolución de una cámara que buscaba sólo la gestión de negocios hacia una visión de rectoría del sector en la zona. Con ello y como ente rector, iniciaron la planificación estratégica de las acciones a futuro de la cámara.

\section{Fortalecer las capacidades de las organizaciones}

1. Para fortalecer a las organizaciones se necesita un proceso de acompañamiento de largo plazo, lo cual a su vez dependerá del nivel de madurez que vayan adquiriendo las organizaciones.

2. En materia de incidencia política, se logró fortalecer espacios de articulación con las instituciones de la comunidad, lo cual se dio por medio de la participación e involucramiento en proyectos específicos, como la celebración del 32 aniversario del PILA,(parque internacional la amistad), la Feria Brunca Emprende y los charlas participativas no programadas. Además, el acercamiento a otras unidades de la UNA como UNA Emprende, da un valor agregado a la labor realizada en la zona Sur, específicamente en el Campus Coto.

\section{RECOMENDACIONES}

A continuación se puntualizan las principales recomendaciones:

1. Se requiere de un equipo de trabajo interdisciplinario para atender las demandas de las organizaciones. Con ello es importante la incorporación de académicos de la sede central como de las regiones a fin de dotar de multidisciplinariedad las acciones de acompañamiento empresarial.

2. El tiempo de ejecución para ciertos proyectos que tienen que ver con asistencia, acompañamiento y extensión rural deben aprobarse en lapsos mayores a un año o a lo sumo dos. Así las cosas, con los tiempos actual y aun cuando se identifica el problema y se propongan las acciones de mejora, se requiere planificación de largo plazo (al menos 5 años) si no más tiempo para su implementación, lo cual no se está logrando.

3. Sobre los Proyectos Funder, la bondad del proyecto es que promueven la incorporación de estudiantes hacia la aplicación práctica de los conocimientos adquiridos en sus carreras.

\section{BIBLIOGRAFIA}

CUEVA, J., GÓMEZ, C., MARTíNEZ, K., ROJAS, H. y YARUQUÍ, A. (2013) Discusión y análisis del documento. Análisis crítico de las teorías de desarrollo económico, Loja, Ecuador, consultado de internet el 22 de febrero 2015, http://es.scribd. com/doc/142175521/Analisis-critico-de-las-teorias-dedesarrollo-economico\#scribd

HERNÁNDEZ MEDINA, C. A. (2007) Universidades y Desarrollo Rural. Papel de las instituciones de educación superior en el diseño de políticas de desarrollo rural, Centro Universitario Municipal Camajuaní, Villa Clara, Cuba. 
MADOERY, O. (2008) Cinco interrogantes fundamentales del desarrollo endógeno, Base de datos EBSCOHOST, recuperado el 20 de abril de 2011.

VARGAS HERNÁNDEZ, J. G. (2007) Desarrollo multinivel: implicaciones macro-regional, local y micro-regional, Instituto Tecnológico de Ciudad Guzmán, Jalisco, México, Provincia N 17, enero-junio 2007, pp. 107-137 I, http:// www.siduna.una.ac.cr:2062/ehost/pdfviewer/pdfview er?vid=6\&hid $=105 \&$ sid $=50 \mathrm{~b} 1448 \mathrm{~d}-5831-4$ a9e-acfef7f25ad57b7f\%40sessionmgr111, consultado el 24 de abril de 2011

WBCSD, World Business Council for Sustainable Development (2002) Negocios para el desarrollo. www.wbcsd.org/web/ development.htm, tomado de este sitio el 20 de abril del 2011. 